\title{
Active tuberculosis, sequelae and COVID-19 co-infection: first cohort of 49 cases
}

\author{
To the Editor:
}

The severe acute respiratory syndrome coronavirus 2 (SARS-CoV-2) disease (COVID-19) pandemic has attracted interest because of its global rapid spread, clinical severity, high mortality rate and capacity to overwhelm healthcare systems $[1,2]$. SARS-CoV-2 transmission occurs mainly through droplets, although surface contamination contributes and debate continues on aerosol transmission [3-5].

The disease is usually characterised by initial signs and symptoms [4-9] similar to those of related viral infections (e.g. influenza, SARS, Middle East respiratory syndrome) and tuberculosis (TB), although prognosis and complications sometimes differ. Experience with concomitant TB and COVID-19 is extremely limited.

One case-control study of COVID-19 patients with interferon- $\gamma$ release assay-confirmed TB infection [10] and a single case of TB with COVID-19 have been submitted to, but not yet published in, peer-reviewed journals [11]. In a recent analysis of 1217 consecutive respiratory specimens collected from COVID-19 patients (Mycobacterium tuberculosis was not tested), the authors concluded that higher rates of co-infection between SARS-CoV-2 and other respiratory pathogens can be expected [12].

The present study describes the first-ever global cohort of current or former TB patients (post-TB treatment sequelae) with COVID-19, recruited by the Global Tuberculosis Network (GTN) in eight countries and three continents. No analysis for determinants of outcome was attempted.

The study is nested within the GTN project monitoring adverse drug reactions $[13,14]$ for which the coordinating centre has an ethics committee approval, alongside ethics clearance from participating centres according to respective national regulation $[13,14]$. A specific nested database was created in collaboration with the eight countries reporting patients with TB and COVID-19; the remaining countries had not yet observed COVID-19 in their patients at the time this manuscript was written.

Continuous variables, if not otherwise specified, are presented as medians with interquartile ranges.

Overall, 49 consecutive patients with current or former TB and COVID-19 from 26 centres in Belgium $(n=1)$, Brazil (Porto Alegre, Rio Grande do Sul State; $n=1$ ), France $(n=12)$, Italy $(n=17)$, Russia (Moscow Region; $\mathrm{n}=6$ ), Singapore $(\mathrm{n}=1)$, Spain $(\mathrm{n}=10)$ and Switzerland (Vaud Canton; $\mathrm{n}=1$ ) were recruited (dataset updated as of 25 April, 2020) (table 1).

The first onset of COVID-19 in the cohort was observed in an Italian patient with TB sequelae on 12 March, 2020 (symptoms from 6 March).

Of 49 patients, 26 (53.0\%) had TB before COVID-19, 14 (28.5\%) had COVID-19 first and nine (18.3\%) had both diseases diagnosed within the same week (four on the same day).

$42(85.7 \%)$ patients had active TB (median age 45.5 (28.0-63.0) years) and seven (14.3\%) had post-TB treatment sequelae (median age $69.0(66.0-70.0)$ years; $\mathrm{p}=0.01$ ); the patients with TB sequelae (from five centres in Italy, Singapore, Spain and Switzerland) were cured 8.2 (2.7-44.3) years earlier.

Overall, 26/49 (53.1\%) patients were migrants, 15/48 (31.3\%) unemployed, and 2/48 (4.1\%) healthcare workers (medical doctor and radiology technician).

46 (93.9\%) patients had confirmed SARS-CoV-2 infection and three other patients (6.1\%) had chest high-resolution computed tomography (HRCT) highly suggestive of COVID-19 related pneumonia (bilateral ground glass opacities) [15].

@ERSpublications

Diagnostic, treatment and outcome details of 49 COVID-19 patients with concurrent or previous tuberculosis from 8 countries show varied clinical profiles https://bit.ly/369ZGGu

Cite this article as: Tadolini M, Codecasa LR, García-García J-M, et al. Active tuberculosis, sequelae and COVID-19 co-infection: first cohort of 49 cases. Eur Respir J 2020; 56: 2001398 [https://doi.org/10.1183/ 13993003.01398-2020]. 
TABLE 1 Demographic, epidemiological and clinical characteristics of a cohort of 49 tuberculosis (TB) patients with coronavirus disease 2019 (COVID-19)

\section{Variable}

\begin{tabular}{|c|c|}
\hline Age years & $48(32-69)$ \\
\hline Male & $40 / 49(81.6 \%)$ \\
\hline Migrant status & $26 / 49(53.1 \%)$ \\
\hline \multicolumn{2}{|l|}{ Occupation } \\
\hline Employed & $14 / 48(29.2 \%)$ \\
\hline Unemployed & $15 / 48(31.3 \%)$ \\
\hline Retired & $14 / 48(29.2 \%)$ \\
\hline Student & $5 / 48(10.4 \%)$ \\
\hline BCG vaccination & $19 / 30(63.3 \%)$ \\
\hline COPD/asthma & $8 / 47(17.0 \%)$ \\
\hline Diabetes mellitus & $8 / 49(16.3 \%)$ \\
\hline HIV infection & $6 / 48(12.5 \%)$ \\
\hline Renal failure & $5 / 49(10.2 \%)$ \\
\hline Liver disease & $7 / 49$ (14.3\%) \\
\hline Alcohol abuse & $10 / 49(20.4 \%)$ \\
\hline Smoking" & $20 / 49(40.8 \%)$ \\
\hline Drug abuse & $4 / 47(8.5 \%)$ \\
\hline \multicolumn{2}{|l|}{ TB } \\
\hline Previous history of TB & $13 / 46(28.3 \%)$ \\
\hline \multicolumn{2}{|l|}{ Site } \\
\hline Pulmonary TB only & $36 / 49(73.5 \%)$ \\
\hline Extrapulmonary TB only & $1 / 49(2.0 \%)$ \\
\hline Pulmonary TB/extrapulmonary TB (>1 site possible) & $12 / 49(24.5 \%)$ \\
\hline \multicolumn{2}{|l|}{ Site of extrapulmonary TB } \\
\hline Bone & $2 / 13(15.4 \%)$ \\
\hline Lymphadenitis & $2 / 13(15.4 \%)$ \\
\hline Pleural & $2 / 13(15.4 \%)$ \\
\hline Central nervous system & $1 / 13(7.7 \%)$ \\
\hline Laryngeal & $1 / 13(7.7 \%)$ \\
\hline Gastrointestinal & $1 / 13(7.7 \%)$ \\
\hline Peritonitis+lymphadenitis+pleural+bone & $1 / 13(7.7 \%)$ \\
\hline Genitourinary+lymphadenitis & $1 / 13(7.7 \%)$ \\
\hline Central nervous system+lymphadenitis & $1 / 13(7.7 \%)$ \\
\hline $\begin{array}{l}\text { Bone+joint+spondylodiscitis (T5-T11)+paravertebral } \\
\text { abscesses }\end{array}$ & $1 / 13(7.7 \%)$ \\
\hline \multicolumn{2}{|l|}{ Radiological involvement } \\
\hline Unilateral cavitary lesions & $10 / 48(20.8 \%)$ \\
\hline Bilateral cavitary lesions & $13 / 48(27.1 \%)$ \\
\hline Bilateral infiltrates (no cavities) & $9 / 48(18.8 \%)$ \\
\hline Unilateral infiltrates & $16 / 48(33.3 \%)$ \\
\hline \multicolumn{2}{|l|}{ Microbiology } \\
\hline Sputum culture confirmation & $22 / 49(44.9 \%)$ (+8 sputum culture ongoing) \\
\hline NAAT confirmation & $13 / 49(26.5 \%)$ \\
\hline Sputum smear positive & $9 / 49(18.4 \%)$ \\
\hline Not available & $5 / 49(10.2 \%)$ \\
\hline \multicolumn{2}{|l|}{ Drug resistance } \\
\hline Resistant & $8 / 45$ (17.8\%) (4 MDR-TB) \\
\hline Sensitive & $37 / 45(82.2 \%)$ \\
\hline \multicolumn{2}{|l|}{ Treatment outcome } \\
\hline Cured & $6 / 49(12.2 \%)$ \\
\hline Completed & $1 / 49(2.0 \%)$ \\
\hline On treatment & $37 / 49(75.5 \%)$ \\
\hline Died $^{+}$ & $5 / 49(10.2 \%)$ \\
\hline Lost to follow-up & 0 \\
\hline Failure & 0 \\
\hline \multicolumn{2}{|l|}{ COVID-19 } \\
\hline \multicolumn{2}{|l|}{ Symptoms } \\
\hline Asymptomatic & $5 / 48$ (10.4\%) \\
\hline Symptomatic & $43 / 48(89.6 \%)$ \\
\hline
\end{tabular}


TABLE 1 Continued

Variable

Imaging

Typical (bilateral ground glass opacities) HRCT picture

Treatment outcome

Resolved

$21 / 44(47.7 \%)$

Still on treatment

$18 / 49(36.7 \%)$

Died lat any datel $)^{+}$

$25 / 49(51.0 \%)$

$6 / 49(12.3 \%)$

Data are presented as number/total number of patients for whom data are available (\%) or median (interquartile range). ${ }^{\#}: \geqslant 14$ drinks per week in men or $\geqslant 7$ drinks per week in women; ": current or ex-smoker; ${ }^{+}$: according to the criteria for cohort analysis of treatment results the number of patients who died during anti-TB treatment is five, but the total number of deaths is six, as one patient with post-TB treatment sequelae died after being cured for TB. BCG: bacillus Calmette-Guérin; NAAT: nucleic acid amplification test; MDR-TB: multidrug-resistant tuberculosis; HRCT: high-resolution computed tomography.

48 patients had pulmonary TB (one caused by Mycobacterium bovis).

37 patients had drug-susceptible (or were treated with the standard first-line regimen for new cases) and eight had drug-resistant TB (and were treated with second-line drugs).

Of the 14 non-clustered patients with COVID-19 diagnosis preceding TB, a child of Gambian origin (3 months old) had SARS-CoV-2 identified 3 days before TB diagnosis although TB was probably pre-existing (pulmonary and extra-pulmonary TB, meningitis). The child is continuing anti-TB treatment and has now recovered from COVID-19. Altogether the diagnosis of COVID-19 preceded that of TB by a median (range) time of 4 (2-10) days.

Those 14 patients, managed in nine centres (in France, Italy, Russia, Spain) were young (median age 33 (26.0-46.0) years); 11/14 (78.5\%) were migrants.

Of the 19 patients undergoing anti-TB treatment, the diagnosis of COVID-19 was made during month 1-2 for 10 (52.6\%), month 3-4 for three (15.7\%), month 4-6 for three (15.7\%) and after 6 months for three (15.7\%) patients.

Signs and symptoms attributed to COVID-19 included (in different combinations) fever (32/48, 81.2\%), dry cough $(27 / 48,56.2 \%)$ and dyspnoea $(17 / 48,35.4 \%)$.

Radiological information was available for 48/49 (98.0\%) patients: 23 (47.9\%) presented cavities (table 1).

21 patients manifested a typical HRCT COVID-19 pattern (bilateral ground glass opacities), whereas 23 had different patterns at HRCT or chest radiography essentially reporting TB-related lesions (infiltrates, consolidations, cavities, etc.) and five were not studied during the course of COVID-19 disease.

$43(87.8 \%)$ patients were hospitalised and, provisionally, the overall median (interquartile range) number of hospital admission days was 15 (8-27). Six patients needed noninvasive ventilation and 14 oxygen supply.

Medication for COVID-19 was reported for 28 patients in all countries except Belgium and Brazil (which had a single case each): 22 (78.6\%) received, in different combinations, hydroxychloroquine, 12 (42.9\%) received an anti-HIV protease inhibitor (i.e. lopinavir/ritonavir, darunavir/cobicistat), seven (25.0\%) patients received azithromycin, and one (3.6\%) patient received other drugs (i.e. enoxaparin and $\mathrm{N}$-acetyl-cysteine (NAC)). 17 (60.8\%) patients received monotherapy (i.e. 11 hydroxychloroquine, five lopinavir/ritonavir and one azithromycin), nine (32.1\%) received a combination of two drugs (i.e. five hydroxychloroquine and protease inhibitor; four hydroxychloroquine plus azithromycin), two (7.1\%) received three or more drugs (i.e. one hydroxychloroquine, lopinavir/ritonavir and azithromycin; one hydroxychloroquine, lopinavir/ritonavir and azithromycin, enoxaparine and NAC).

The case fatality rate was high $(6 / 49,12.3 \%)$; five of these six were $>60$ years old and all of them had at least one comorbidity. Given the small number of deaths, larger studies are necessary.

This preliminary analysis suggests that:

1) In 19/49 (38.8\%) patients, COVID-19 appeared during anti-TB treatment and limited or no protection against COVID-19 might have favoured SARS-CoV-2 infection (which affected two healthcare workers). 
2) The diagnosis of TB and COVID-19 was done simultaneously or within 7 days in nine patients, posing differential diagnosis challenges, suggesting that clinical assessments to investigate COVID-19 (e.g. clinical picture, HRCT) facilitated the identification of (a probably pre-existing) TB. Any contribution of COVID-19 to TB pathogenesis cannot be excluded or confirmed.

3) Although the diagnosis of COVID-19 preceded that of TB in 14 patients, larger studies are needed to understand any role played by SARS-CoV-2 in the progression of TB infection to disease. Given that up to a quarter of the population in some of the regions studied are latently infected, SARS-CoV-2 infection might boost the development of active TB in the coming months [10]. As we do not include individuals with latent TB infection followed up over time, we cannot report on the potential contribution of COVID-19 towards development of active disease. Probably, an overlap of signs/ symptoms of COVID-19 and TB occurred, and COVID-19 was diagnosed earlier because of a higher index of suspicion, while TB may have been previously present. Or, differently, COVID brought to clinical evaluation/diagnostic assessment $\mathrm{TB}$ patients at an earlier stage of disease, before the occurrence of TB-related symptoms.

4) In seven cases, COVID-19 occurred in patients with $\mathrm{TB}$ sequelae. They were older than patients under anti-TB treatment and presented higher (although not statistically significant) mortality. All but one had comorbidities (four COPD; one HIV co-infection plus liver and kidney diseases, hypertension and cancer present in different combinations). They presented unilateral or bilateral radiological sequelae of previous infiltrates (four patients) or cavities (three patients). Larger numbers are necessary to further understand the role played by TB sequelae.

5) The impact on the healthcare system (days of admission, intensive care unit beds, etc.) was relevant, and will deserve further evaluation.

6) The information on BCG (bacillus Calmette-Guérin) vaccination is modest (30 patients with information, 19 previously vaccinated in all eight countries) and no significant elements can be provided to the ongoing debate on its protective role.

7) We presently have no data on drug-drug interactions.

This is, to our knowledge, the first published cohort of patients with active TB and COVID-19. Our study represents a "snapshot" of a cohort of patients at different stages of disease. No attempt was made to obtain representation of the larger universe of patients with both diseases and the small sample size precludes an analysis of risk factors. We cannot exclude that some findings have a casual origin.

The information available on the patients recently admitted was accurate, but some details on previous TB were incomplete, and some examinations were not performed either because the patients refused or the patients' condition was too severe. Although case reporting is comprehensive in the countries/regions covered by GTN, the study reflects the initial stages of the COVID-19 epidemic only, and representative longitudinal observations will be necessary to evaluate the interactions between COVID-19 and TB.

We believe that this descriptive research can motivate larger studies to enable analyses of interactions and determinants of outcomes in patients with both diseases. The study will continue to follow-up the patients and accrue more records. We therefore invite interested clinicians and programmes to contact the corresponding author and help improve the understanding of how to optimise care for these patients.

Marina Tadolini ${ }^{1,44}$, Luigi Ruffo Codecasa ${ }^{2,44}$, José-María García-García $\odot^{3}$, François-Xavier Blanc $\oplus^{4}$, Sergey Borisov ${ }^{5}$, Jan-Willem Alffenaar ${ }^{6,7,8}$, Claire Andréjak ${ }^{9}$, Pierre Bachez ${ }^{10}$, Pierre-Alexandre Bart ${ }^{11}$, Evgeny Belilovski ${ }^{5}$, José Cardoso-Landivar ${ }^{12}$, Rosella Centis $\odot^{13}$, Lia D'Ambrosio $\odot^{14}$, María-Luiza De Souza-Galvão ${ }^{12}$, Angel Dominguez-Castellano ${ }^{15}$, Samir Dourmane ${ }^{16}$, Mathilde Fréchet Jachym ${ }^{17}$, Antoine Froissart ${ }^{18}$, Vania Giacomet ${ }^{19}$, Delia Goletti ${ }^{20}$, Soazic Grard ${ }^{21}$, Gina Gualano ${ }^{22}$, Armine Izadifar ${ }^{23}$, Damien Le Du ${ }^{17}$, Margarita Marín Royo ${ }^{24}$, Jesica Mazza-Stalder ${ }^{25}$, Ilaria Motta ${ }^{26}$, Catherine Wei Min Ong ${ }^{27,28}$, Fabrizio Palmieri $\oplus^{22}$, Frédéric Rivière ${ }^{29}$, Teresa Rodrigo $\oplus^{3}$, Denise Rossato Silva $^{30}$, Adrián SánchezMontalvá ${ }^{31,32,33}$, Matteo Saporiti ${ }^{2}$, Paolo Scarpellini $\oplus^{34}$, Frédéric Schlemmer $\oplus^{35}$, Antonio Spanevello ${ }^{36,37}$, Elena Sumarokova ${ }^{5}$, Eva Tabernero ${ }^{38}$, Paul Anantharajah Tambyah ${ }^{27,28}$, Simon Tiberi $\overbrace{}^{39,40}$, Alessandro Torre ${ }^{41}$, Dina Visca $\oplus^{36,37}$, Miguel Zabaleta Murguiondo ${ }^{42}$, Giovanni Sotgiu $\oplus^{43}$ and Giovanni Battista Migliori $\circledast^{13}$

${ }^{1}$ Unit of Infectious Diseases, Dept of Medical and Surgical Sciences, Alma Mater Studiorum University of Bologna, Bologna, Italy. ${ }^{2}$ TB Reference Centre, Villa Marelli Institute, Niguarda Hospital, Milan, Italy. ${ }^{3}$ Tuberculosis Research Programme (PII-TB), SEPAR, Barcelona, Spain. ${ }^{4}$ Centre Hospitalier Universitaire, Nantes, France. ${ }^{5}$ Moscow Research and Clinical Center for TB Control, Moscow, Russian Federation. ${ }^{6}$ The University of Sydney, Sydney Pharmacy School, Sydney, Australia. ${ }^{7}$ Westmead Hospital, Sydney, Australia. ${ }^{8}$ Marie Bashir Institute of Infectious Diseases and Biosecurity, University of Sydney, Sydney, Australia. ${ }^{9}$ Service de Pneumologie CHU AMIENS PICARDIE, France AND UR Université de Picardie Jules Verne, Amiens, France. ${ }^{10}$ Service de Pneumologie, Clinique Saint Luc, Bouge, Belgium. ${ }^{11}$ Dept of Internal Medicine, Lausanne University, Lausanne, Switzerland. ${ }^{12}$ Servicio Neumología, Vall D'Hebron University Hospital, Barcelona, Spain. ${ }^{13}$ Servizio di Epidemiologia Clinica delle Malattie Respiratorie, Istituti Clinici Scientifici Maugeri IRCCS, Tradate, Italy. ${ }^{14}$ Public Health Consulting Group, Lugano, Switzerland. ${ }^{15}$ Servicio de Enfermedades Infecciosas y Microbiología, Hospital Virgen Macarena, Sevilla, Spain. ${ }^{16}$ Service de Pneumologie, Groupe hospitalier sud île de France (GHSIF), Melun, France. ${ }^{17}$ Centre Hospitalier de Bligny, Briis Sous Forges, France. ${ }^{18}$ Service de Médecine interne, CHI de Créteil, Créteil, France. ${ }^{19}$ Pediatric Infectious Diseases Unit, Dept of Biomedical and Clinical Sciences, L. Sacco Hospital, University of Milan, Milan, Italy. ${ }^{20}$ Translational Research Unit, National 
Institute for Infectious Diseases 'L. Spallanzani', IRCCS, Rome, Italy. ${ }^{21}$ Centre de Lutte Antituberculeuse (CLAT 38), Grenoble, France. ${ }^{22}$ Respiratory Infectious Diseases Unit, National Institute for Infectious Diseases 'L. Spallanzani', IRCCS, Rome, Italy. ${ }^{23}$ Hôpital Européen de Paris La Roseraie, Aubervilliers, France. ${ }^{24}$ Servicio Neumología, Hospital General Universitario de Castellón, Castelló, Spain. ${ }^{25}$ Pulmonary Division, Lausanne University Hospital CHUV, Centre Hospitalier Universitaire Vaudois, Lausanne, Switzerland. ${ }^{26}$ Dipartimento di Scienze Mediche, Clinica Universitaria Malattie Infettive, Ospedale Amedeo di Savoia, Torino, Italia. ${ }^{27}$ Dept of Medicine, Yong Loo Lin School of Medicine, National University of Singapore, Singapore. ${ }^{28}$ Institute for Health Innovation and Technology (iHealthtech), National University of Singapore, Singapore. ${ }^{29}$ Hôpital d'Instruction des Armées (HIA) Percy, Clamart, France. ${ }^{30}$ Faculdade de Medicina, Universidade Federal do Rio Grande do Sul - UFRGS, Porto Alegre, Brazil. ${ }^{31}$ Infectious Diseases Dept, International Health and Tuberculosis Unit, Vall d'Hebron University Hospital, Barcelona, Spain. ${ }^{32}$ Vall d'Hebron Institute of Research (VHIR), Barcelona, Spain. ${ }^{33}$ Grupo de Estudio de Infecciones por Micobacterias (GEIM), Spanish Society of Infectious Diseases (SEIMC), Spain. ${ }^{34}$ Unit of Infectious Diseases, Università Vita e Salute, San Raffaele Hospital, Milan, Italy. ${ }^{35}$ Hôpitaux Universitaires Henri Mondor, AP-HP, Créteil, France. ${ }^{36}$ Division of Pulmonary Rehabilitation, Istituti Clinici Scientifici Maugeri, IRCCS, Tradate, Italy. ${ }^{37}$ Dept of Medicine and Surgery, Respiratory Diseases, University of Insubria, Tradate, Italy. ${ }^{38}$ Servicio Neumología, Hospital de Cruces, Bilbao, Spain. ${ }^{39}$ Blizard Institute, Barts and The London School of Medicine and Dentistry, Queen Mary University of London, London, UK. ${ }^{40}$ Division of Infection, Royal London Hospital, Barts Health NHS Trust, London, UK. ${ }^{41}$ Dept of Infectious Diseases, University of Milan, L. Sacco Hospital, Milan, Italy. ${ }^{42}$ Servicio Neumología Hospital Universitario Marqués de Valdecilla, Santander, Spain. ${ }^{43}$ Clinical Epidemiology and Medical Statistics Unit, Dept of Medical, Surgical and Experimental Sciences, University of Sassari, Sassari, Italy. ${ }^{44}$ Co-first authors.

Correspondence: Giovanni Battista Migliori, Servizio di Epidemiologia Clinica delle Malattie Respiratorie, Istituti Clinici Scientifici Maugeri IRCCS, Via Roncaccio 16, Tradate, Varese, 21049, Italy. E-mail: giovannibattista.migliori@icsmaugeri.it

Received: 25 April 2020 | Accepted after revision: 10 May 2020

This article has an editorial commentary: https://doi.org/10.1183/13993003.01348-2020

Acknowledgements: The article is part of the scientific activities of the Global Tuberculosis Network (GTN); GREPI (Groupe de Recherche et d'Enseignement en Pneumo-Infectiologie), a working group from SPLF (Société de Pneumologie de Langue Française); SEPAR (Sociedad Española de Neumología and Cirugía Torácica); Moscow Society of Phtisiology and of the WHO Collaborating Centre for Tuberculosis and Lung Diseases, Tradate, ITA-80, 2017-2020GBM/RC/LDA). Delia Goletti is a professor of Pathology at Unicamillus University in Rome, Italy. The authors wish to thank Enrico Girardi (National Institute for Infectious Diseases, L. Spallanzani, IRCCS, Rome, Italy) for the useful comments on the manuscript.

Conflict of interest: None declared.

\section{References}

1 Worldometers. COVID-19 Coronavirus Pandemic. www.worldometers.info/coronavirus Date last updated: April 23,2020 .

2 Vitacca M, Nava S, Santus P, et al. Early consensus management for non-ICU ARF SARS-CoV-2 emergency in Italy: from ward to trenches. Eur Respir J 2020; 55: 2000632.

3 Lewis D. Is the coronavirus airborne? Experts can't agree. Nature 2020; 580: 175.

4 Leung CC, Lam TH, Cheng KK. Let us not forget the mask in our attempts to stall the spread of COVID-19. Int J Tuberc Lung Dis 2020; 24: 364-366.

5 Esposito S, Principi N, Leung CC, et al. Universal use of face masks for success against COVID-19: evidence and implications for prevention policies. Eur Respir J 2020; 55: 2001260.

6 Guan WJ, Ni ZY, Hu Y, et al. Clinical characteristics of coronavirus disease 2019 in China. N Engl J Med 2020; 382: $1708-1720$,

7 Xu XW, Wu XX, Jiang XG, et al. Clinical findings in a group of patients infected with the 2019 novel coronavirus (SARS-Cov-2) outside of Wuhan, China: retrospective case series. BMJ 2020; 368: m606.

8 Zhou F, Yu T, Du R, et al. Clinical course and risk factors for mortality of adult inpatients with COVID-19 in Wuhan, China: a retrospective cohort study. Lancet 2020; 395: 1054-1062.

9 Noia Maciel EL, Gonçalves Júnior E, Pretti Dalcolmo MM. Tuberculosis and coronavirus: what do we know? Epidemiol Serv Saúde 2020; 29: e2020128.

10 Liu Y, Bi L, Chen Y, et al. Active or latent tuberculosis increases susceptibility to COVID-19 and disease severity. MedRxiv 2020; preprint [https://doi.org/10.1101/2020.03.10.20033795].

11 Singh A, Gupta A, Das K. Severe acute respiratory syndrome coronavirus-2 and pulmonary tuberculosis coinfection: double trouble. Research Square 2020; preprint [https://doi.org/10.21203/rs.3.rs-22464/v1].

12 Kim D, Quinn J, Pinsky B, et al. Rates of co-infection between SARS-CoV-2 and other respiratory pathogens. JAMA 2020; 323: 2085-2086.

13 Borisov S, Danila E, Maryandyshev A, et al. Surveillance of adverse events in the treatment of drug-resistant tuberculosis: first global report. Eur Respir J 2019; 54: 1901522.

14 Akkerman O, Aleksa A, Alffenaar JW, et al. Surveillance of adverse events in the treatment of drug-resistant tuberculosis: A global feasibility study. Int J Infect Dis 2019; 83: 72-76.

15 Wen Z, Chi Y, Zhang L, et al. Coronavirus disease 2019: initial detection on chest CT in a retrospective multicenter study of 103 Chinese subjects. Radiol Cardiothorac Imag 2020; 2: e200092 